\title{
Phenolics composition in lettuce upon ultraviolet radiation and temperature effects
}

\author{
Oksana SYTAR ${ }^{1,2}$ - Marek ZIVCAK ${ }^{1}$ - Marian BRESTIC ${ }^{1}$ - Cornelia RAUH ${ }^{3}-$ \\ Ivan $\mathrm{SIMKO}^{4}$ \\ 1: Department of Plant Physiology, Slovak University of Agriculture, Nitra, A. Hlinku 2, 94976 Nitra, Slovak \\ Republic \\ 2: Departement of Plant Biology, Educational and Scientific Center 'Institute of Biology', TarasShevchenko \\ National University of Kyiv, Kyiv, Ukraine \\ 3: Department of Food Biotechnology and Food Process Engineering, Institute of Food Technology and Food \\ Chemistry TU Berlin, Königin-Luise-Str., 22, 14195 Berlin, Germany \\ 4: U.S. Department of Agriculture, Agricultural Research Service, U.S. Agricultural Research Station, Crop \\ Improvement and Protection Research Unit, 1636 E. Alisal St., Salinas, CA 93905, USA
}

Keywords: flavonoids, phenolic acids, environmental stresses, high temperature, pigments

\section{Introduction}

The accumulation of secondary metabolites in plants depends on both external (environmental) and internal (biological) factors. Although, the variation observed among cultivars is generally considered to be more important than environmental effects. We have previously demonstrated that fast, fluorescence record analyzed by the fluorescence excitation ratio method (Cerovic et al., 2002) can efficiently determine environmentally induced changes in the content of flavonoids and anthocyanins in lettuce leaves with different colors. The aim of the present study was to test the hypotheses that (1) the accumulation of UV-absorbing and other related phenolic compounds responds specifically to altered UV radiation and temperature; (2) the differences exist in the accumulation of phenolic compounds between green-colored and red-colored lettuce cultivars when exposed to different environmental conditions; (3) the non-destructive quantifications of flavonoids and phenolics using the fluorescence excitation ratio method can produce the data consistent with the results of standard analytical methods; and (4) there is a close relationship between the content of specific phenolic acids and major groups of polyphenols.

\section{Material and methods}

The experiments were held at SAU in Nitra $\left(48^{\circ} 19^{\prime} 7^{\prime \prime} \mathrm{N}, 18^{\circ} 4^{\prime} 55^{\prime \prime} \mathrm{E}, 144 \mathrm{~m}\right.$ asl). To distinguish the effects of UV radiation from other environmental factors such as temperature, humidity, and light intensity, the plants were grown in three different environments: 1 . direct sunlight (outdoor conditions with high UV), 2. under clear glass (outdoor conditions with low UV), and 3. greenhouse (indoor conditions with low UV) Measurements of chlorophyll fluorescence under the multiple light spectra excitations were done using the portable optical fluorescence sensor Multiplex-3® (Force-A, Paris, France). Total phenolic content from the lettuce leaves extracts was determined by the standard spectrophotometric method using Folin-Ciocalteu reagent (Singleton and Rossi, 1965). Hydroxycinnamic acid derivatives were quantified using the approach of Mewis et al. (2011). 


\section{Results and discussion}

Environmental conditions influence the content and metabolic profile of phenolic compounds in vegetables. The present study focused on distinguishing between the effects of ultraviolet (UV) radiation and temperature on lettuce plants with green or red leaf color when grown in either a greenhouse or outdoors. A combination of the non-destructive, fluorescence-based method with spectrophotometric and HPLC analyses enabled us to assess the effects of environment, cultivar, and plant-leaf color on concentrations of flavonoids, anthocyanins, and phenolic compounds. The accumulation of total phenolics, flavonoids, anthocyanins, and phenolic acids (benzoic acid derivatives and cinnamic acid derivatives) increased in direct sunlight (high UV radiation, moderate temperature) conditions outdoors as compared to the greenhouse conditions (low UV radiation, high temperature).

The comparison of the effects of UV radiation and temperature (indoor-outdoor effect) indicated that the level of UV radiation plays a dominant role in the accumulation of flavonoids, anthocyanins, and methoxycinnamic acid; while temperature predominantly influences the accumulation of phenolic acids (rosmarinic, p-anisic, vanillic acid. Although, the leaf color (green vs. red) was strongly related to the content of majority of studied phytochemicals, environmental conditions affected their concentrations in both color types similarly. The concentrations of compounds estimated with the noninvasive, fluorescence excitation ratio method was highly consistent with those obtained by standard analytical approaches. Our results show that this fast, non-invasive method can be effectively used for determining concentrations of flavonoids and phenolic acids in lettuce plants.

\section{Acknowledgement}

This work was supported by the research project of the Scientific Grant Agency of Slovak Republic VEGA- 1-0923-16, VEGA-1-0831-17 and APVV-15-0721.

\section{References}

Cerovic, Z.G., Ounis, A., Cartelat, A., Latouche, G., Goulas, Y., Meyer, S., Moya, I. (2002): The use of chlorophyll fluorescence excitation spectra for the non-destructive in situ assessment of UV-absorbing compounds in leaves. Plant. Cell. Environ. 25:. 1663-1676. doi:10.1046/j.1365-3040.2002.00942.x

Mewis, I., Smetanska, I.M., Müller, C.T., Ulrichs, C. (2011): Specific poly-phenolic compounds in cell culture of Vitis vinifera L. cv. Gamay Fréaux. Appl. Biochem. Biotechnol. 164. 148-161. doi: 10.1007/s12010-0109122-x

Singleton, V.L., Rossi, J.A. (1965): Colorimetry of total phenolics with phosphomolybdicphosphotungstic acid reagents. Am. J. Enol. Vitic. 16. 144-158. 